Case report

\title{
Cerebral alveolar echinococcosis: A report of two cases
}

\author{
Zhan-Long $\mathrm{Ma}^{\mathrm{a}, *}$, Li-Gong $\mathrm{Ma}^{\mathrm{b}}$, Yicheng $\mathrm{Ni}^{\mathrm{c}}$ \\ a Department of Radiology, Yangzhou No. 1 People's Hospital, Yangzhou, China \\ b Department of Radiology, Affiliated Hospital, Qinghai University, Xining, China \\ c Department of Radiology, University Hospitals, Catholic University of Leuven, Belgium
}

\section{A R T I C L E I N F O}

\section{Article history:}

Received 3 December 2010

Received in revised form 28 October 2011

Accepted 2 December 2011

Available online $\mathrm{xxx}$

\section{Keywords:}

Brain

Alveolar echinococcosis

Computed tomography

Magnetic resonance imaging

Cerebral alveolar echinococcosis is relatively rare and its clinical diagnosis is often difficult [1]. Computed tomography (CT) and magnetic resonance imaging (MRI) are helpful in the diagnosis of cerebral alveolar echinococcosis [2]. In the following report, we describe the clinical, radiological and histopathological features of this disease in two patients that we have encountered recently, which is complemented with a brief literature review.

\section{Case report}

Patient 1 (Fig. 1a-e): A 50-year-old man presented with persisting headache, nausea and vomiting for two weeks, which worsened in the last two days. There was a history of prior living in sheepraising hydatid endemic areas.

Brain CT demonstrated two calcified lesions surrounded by edema in the straight gyrus of frontal lobe and left temporal lobe respectively (Fig. 1a). Subsequently MRI (0.5T, Philips) was performed. On $\mathrm{T}_{2}$-weighted images, lesions showed a markedly low signal corresponding to central calcification with extensive peripheral high signals suggestive of surrounding edema (Fig. 1b). On precontrast $\mathrm{T}_{1}$-weighted MRI, the lesions appeared as hypointense and heterogeneous beehive-like structures with ill-defined border (Fig. 1c). After gadolinium injection, the lesions demonstrated

\footnotetext{
* Corresponding author at: Department of Radiology, Yangzhou No. 1 People's Hospital, 368\# Hangjiang Road, Yangzhou 225001, China. Tel.: +86 51482981199; fax: +86 51482981199 .

E-mail address: mazhanlong808@sina.com (Z.-L. Ma).
}

cauliflower-like contrast enhancement (Fig. 1d). Based on a positive result from the Casoni test, which is a skin test used in the diagnosis of hydatid disease involving the intradermal injection of sterilised fluid from hydatid cysts with a wheal response occurring within 20 min being considered positive, a surgical treatment of the lesions was performed, and histopathology suggested a diagnosis of cerebral alveolar echinococcosises (Fig. 1e). Receiving the treatment with parasitostatic medication (albendazole), this patient showed no recurrence of the lesion during 2 years of follow up.

Patient 2(Fig. 2a-g): A 42-year-old woman was referred to us for imaging examinations by an oncology clinic because of her clinical deteriorations. She had been complaining of headache and vomiting for the preceding five days. Noninvasive imaging investigations revealed a hepatic mass as well as two cerebral masses. She had the history of living in hydatid disease endemic areas for about 5 years.

Plain CT demonstrated a mass of $8 \mathrm{~cm} \times 6 \mathrm{~cm}$ in the right lobe of the liver with clustered hyperdense areas of central calcification and vague irregular borders (Fig. 2a). After intravenous injection of contrast media, the mass could not be enhanced in the arterial phase but with better delineation due to strong liver enhancement (Fig. 2b).

Cerebral CT (Fig. 2c) showed a well-defined mass consisting of hyperdense calcifications and central necrosis in the left brain (thick arrow). Another smaller hyperdense mass was also showed under the dura on the left (thin arrow).

Cerebral MRI demonstrated two intraparenchymal masses: one in the left frontal lobe and the other under the dura of the left temple lobe (Fig. 2d-f). On T1-weighted plain images, the two lesions were slightly hypointense relative to the white matter with 

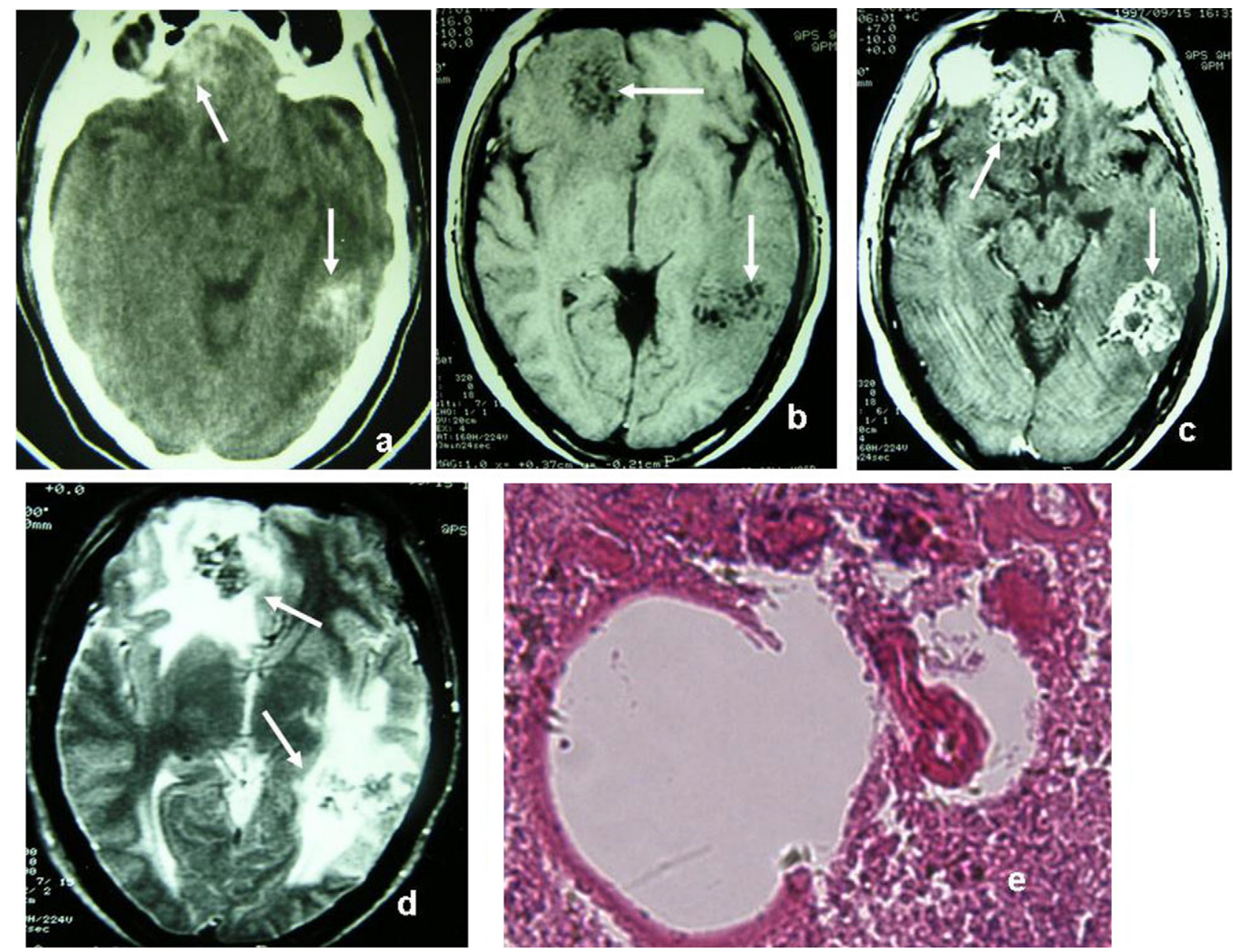

Fig. 1. Cerebral alveolar echinococcosises.

the surrounding areas of even lower signal intensities (Fig. 2d). After injection of gadolinium contrast agent, the lesions were cauliflower-like enhanced (Fig. 2e). T2-weighted image revealed the two heterogeneous hypointense masses with hyperintense surrounding areas consistent with edema around the calcification (Fig. 2f). The Casoni test was positive. After hepatic lobectomy for surgical treatment of the cerebral lesion, the disease was confirmed by histopathological examinations as a hepatic alveolar echinococcosises with metastases to the brain (Fig. 2g). With chemotherapy using parasitostatic medication (albendazole), this patient remained no neurological symptom for 2 years of follow-up.

\section{Discussion}

The hydatid disease has been diagnosed most frequently in the endemic areas such as Central Europe, Russia, China, North America, Turkey, Australia, New Zealand and the Mediterranean [1,2].

Hydatid disease is caused by the metacestode form of Echinococcus multilocularis. The liver (50-77\%) and the lung (8.5-43\%) are the organs most commonly involved [3]. In humans, there are two main forms of hydatid disease that are cycstic echinococcosis, caused by Echinococcus granulosus, and less frequently alveolar echinococcosis (AE), caused by Echinococcus multilocularis [4,5]. Cerebral occurrence of $\mathrm{AE}$ is rare, accounting for only $1 \%$ of the patients with hepatic $\mathrm{AE}$, and is generally considered to be fatal [6].
Alveolar echinococcosis diseases are benign with slow growing pattern and randomly located spreading lesions. The cerebral lesions may remain asymptomatic until they become quite large. Headache and vomiting are the most common symptoms in other reported series as well as in our patients. Other symptoms such as hemiparesis, seizures, visual field alteration and gait disorders, varying with the intracranial location of the disease, are nonspecific clinical features [7].

The exogenous budding alveolar cysts infiltrate the liver diffusely rather than forming a solitary cyst as does Echinococcus granulosus [8]. In the brain, the cyst of alveolar echinococcosis also differs from that of cystic echinococcosis in that it grows by extending or budding of the germinal membrane with progressive infiltration into the surrounding tissue. Currently, surgery is the accepted method for the treatment of cerebral AE cases but has to be followed by chemotherapy.

The CT and MRI appearances of cerebral alveolar echinococcosis have rarely been reported. On CT and MRI, the lesions of cerebral alveolar echinococcosis appear as a solid, semisolid, or multilocular cystic mass with definite margins. Calcification and surrounding edema are common features. Contrast enhancement occurs within the region of inflammatory reaction around the cystic mass [9]. In our cerebral alveolar echinococcosis cases, CT and MRI also demonstrated calcified, round, solid lesions with contrast enhancement and surrounding edema, though these features were not specific for the AE diagnosis. In our cases, the cerebral AE lesions showed cauliflower-like contrast enhancement. This 

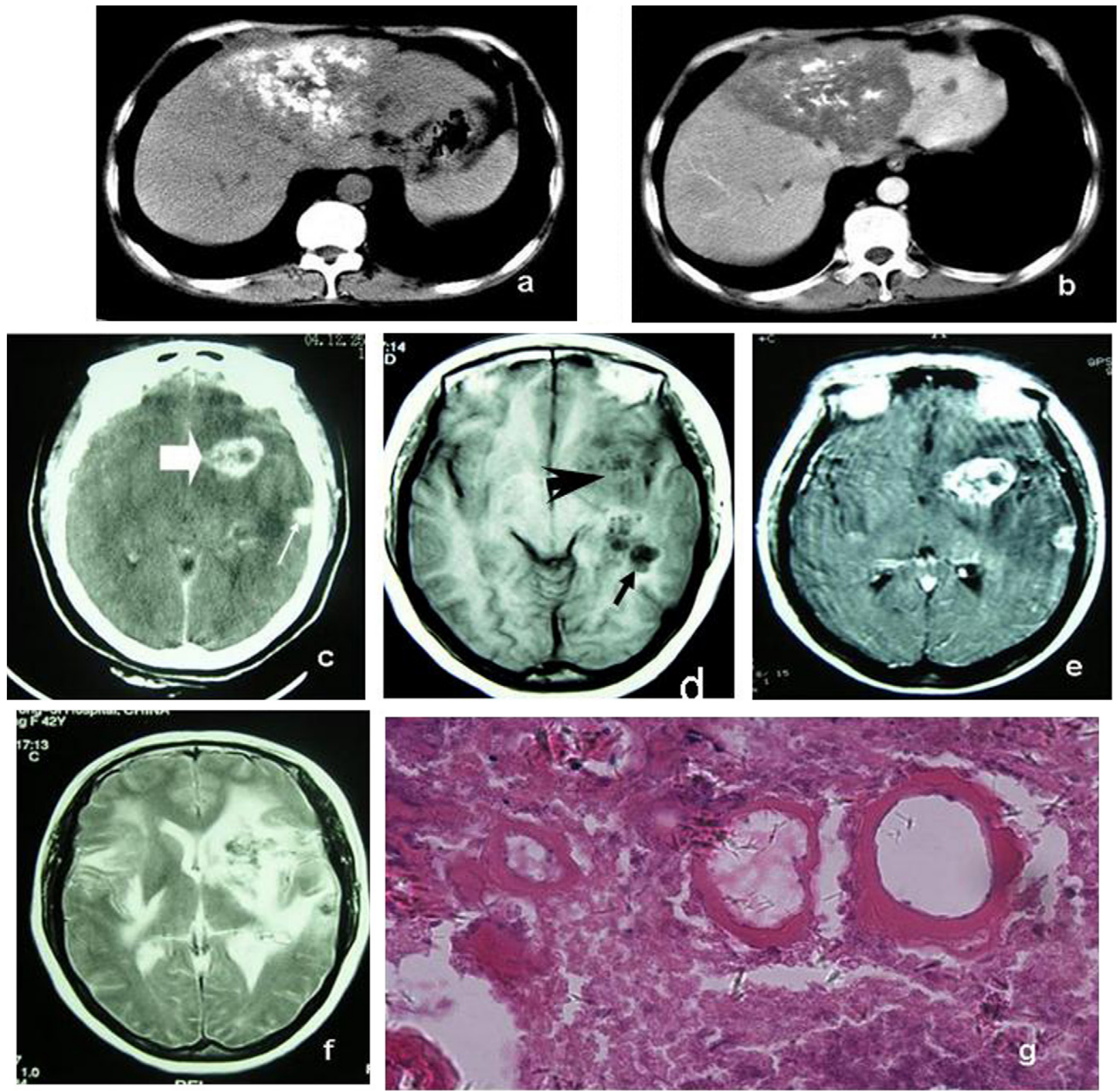

Fig. 2. Hepatic alveolar echinococcosises with metastases to the brain.

pattern was also reported from other's study [10,11], which may help distinguish the cerebral AE from other brain masses, particularly cysticercosis, coenuresis, toxoplasmosis, fungal infections, brain abscesses and oligodendrogliomas.

The differential diagnosis of cerebral alveolar echinococcosis includes gliomas, metastases, tuberculomas [11-13]. The diagnosis should be suggested by evidence of primary hepatic and cerebral focus, appropriate clinical history, prevalence of the infection in the host's geographic location, and laboratory findings. Besides, the information about CSF, Western blot or PCR should be acquired before surgery and as a standard practice for diagnosis and differential diagnosis of cerebral AE.

The prevalence of $\mathrm{AE}$ is low and the occurrence of this disease is geographically restricted. Nevertheless, due to travel and immigration, it is imperative that radiologists and clinicians understand the typical distribution, patterns, and imaging manifestations of cerebral alveolar echinococcosis lesions.

\section{References}

[1] Craig PS, Echinococcosis Working Group in China. Epidemiology of human alveolar echinococcosis in China. Parasitol Int 2006:S221-5 [Suppl].

[2] Eckert J, Deplazes P. Biological, epidemiological, and clinical aspects of echinococcosis, a zoonosis of increasing concern. Clin Microbiol Rev 2004; 17(1):107-35.

[3] Tuzun M, Altinors N, Arada IS, et al. Cerebral hydatid disease CT and MR findings. Clin Imaging 2002;26:353-7.

[4] Beggs I. The radiology of hydatid disease. Am J Roentgenol 1985;145:639-48.

[5] Aydinli B, Aydin U, Yazici P, et al. Alveolar echinococcosis of liver presenting with neurological symptoms due to brain metastases with simultaneous lung metastasis: a case report. Turkiye Parazitol Derg 2008;32(4):371-4.

[6] Algros MP, Majo F, Bresson-Hadni S, et al. Intracerebral alveolar echinococcosis. Infection 2003;31(1):63-5. 
[7] El-Shamam O, Amer T, El-Atta MA. Magnetic resonance imaging of simple and infected hydatid cysts of the brain. Magn Reson Imaging 2001;19:965-74.

[8] Lafond DJ, Thatcher DS, Handeyside RG. Alveolar hydatid disease. JAMA 1963;186:35-7.

[9] Topsakal C, Aydin Y, Sahin Y, et al. Cerebral alveolar hydatidosis: case report. Surg Neurol 1996;45:575-81.

[10] Tunaci M, Tunaci A, Engin G, et al. MRI of cerebral alveolar echinococcosis. Neuroradiology 1999;41(8):844-6.
[11] Piotin M, Cattin F, Kantelip B, et al. Disseminated intracerebral alveolar echinococcosis: CT and MRI. Neuroradiology 1997;39(6):431-3.

[12] Senturk S, Oquz KK, Soylemezoqlu F, et al. Cerebral alveolar echinococcosis mimicking primary brain tumor. Am J Neuroradiol 2006;27(2):420-2.

[13] Ozdol C, Yildirim AE, Daglioglu E, Divanlioglu D, Erdem E, Belen D. Alveolar hydatid cyst mimicking cerebellar metastatic tumor. Surg Neurol Int $2011 ; 29(2): 13$ 